\begin{tabular}{|c|c|}
\hline \multirow{3}{*}{ 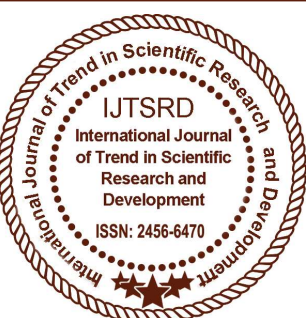 } & $\begin{array}{l}\text { International Journal of Trend in Scientific } \\
\text { Research and Development (IJTSRD) }\end{array}$ \\
\hline & International 0 \\
\hline & ISSN No: 2456 - 6470 | www.ijtsrd.com | Volume - 2 | Issue - 5 \\
\hline
\end{tabular}

\title{
Performance Analysis of V-Blast MIMO System Using Minimum Mean Square Error Equalizer Technique with BPSK
}

\author{
Tamashri Sonartiya ${ }^{1}$, Deepak Pancholi ${ }^{2}$ \\ M.Tech Student ${ }^{1}$, Assistant Professor ${ }^{2}$ \\ Department of Electronics and Communication Engineering \\ Lakshmi Narain College of Technology, Indore, Madhya Pradesh, India
}

\section{ABSTRACT}

The V-BLAST MIMO-system some of linear detection techniques can be used for interference cancellation (IC). In this paper, using Minimum Mean Square Error- interference cancellation for the same. In this paper, we analysis of BER performance of Vertical Bell Labs Layered Space Time Architecture (V-BLAST) spatial Multiplexing Technique with equalisation techniques like Minimum Mean Square Error (MMSE) by BPSK modulation techniques in Rayleigh flat fading channel.

Keywords: V-Blast, BPSK, MMSE, Equalizer, interference cancellation, Rayleigh Channel.

\section{INTRODUCTION}

The BLAST architecture, introduced by Foschini [12] in 1996, is a low complexity transceiver architecture to communicate over the MIMO wireless channel. Although suboptimal, it is able to attain a significant fraction of the theoretical MIMO capacity over the rich-scattering wireless channel. BLAST implies independent transmission of streams at the transmitter side and successive interference cancellation (SIC) at the receiver. In 1998, V-BLAST (Vertical BLAST) was introduced in [2] as a low complexity wireless communication architecture. In the V-BLAST, the layering is horizontal, meaning that all the symbols of a certain stream are transmitted through the same antenna (one stream per antenna). It was shown in [3] that this architecture is able to achieve very high spectral efficiencies e.g. spectral efficiencies in the order of $20-40 \mathrm{bits} / \mathrm{sec} / \mathrm{Hz}$ in an indoor propagation environment at realistic SNR's and error rates.

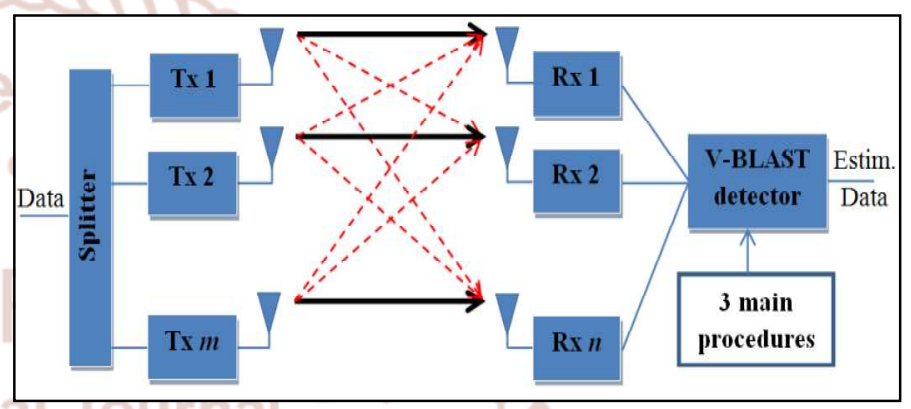

Fig. 1: V-Blast Algorithms

\section{EQUALIZER TECHNIQUE}

Equalization is the technique which is used to mitigate the effect of the ISI (inter symbol interference) by minimizing the error probability occur in the communication system without ISI suppression method. Since, suppression of ISI causes the noise power to enhance therefore it is essential to create optimum balance between enhancement of noise power and suppression of ISI [6], one of the practical problems in digital communications is inter-symbol interference (ISI), which causes a given transmitted symbol to be distorted by other transmitted symbols. One of the most commonly used techniques to counter the channel distortion (ISI) is linear channel equalization. Conventional equalization techniques employ a pre-assigned time slot (periodic for the timevarying situation). In the receiver the equalizer coefficients are then changed (e.g. LMS, MMSE, etc.).

\section{A. MMSE Equalizer}

This type of equalizer applies the squared error for performance measurement. The receiver filter is designed and develops to satisfy the minimum mean square error criterion. Main objective of this 
technique is to minimize the error produced between target signal and output obtained by filter $[6,10]$.

\section{COMMUNICATION CHANNEL}

Due to the multipath characteristic of the wireless propagation channel, multiple copies of the transmitted signal arrive at the receiver at different moments of time. This combination of signals with a phase difference at the receiver causes abrupt variations in the received signal power or in the received SNR, which is known as fading. The NLOS (indoor, city) Rayleigh fading occurs when there is no multipath LOS between transmitter and receiver and have only indirect path which is called NLOS to receive the resultant waves. The Rayleigh Fading is one kind of model which propagates the environment of radio signal. Rayleigh fading works as a reasonable model when many objects in environment which scatter radio signal before arriving of receiver. When there is no propagation dominant during line of sight between transmitter and receiver on that time Rayleigh Fading is most applicable.

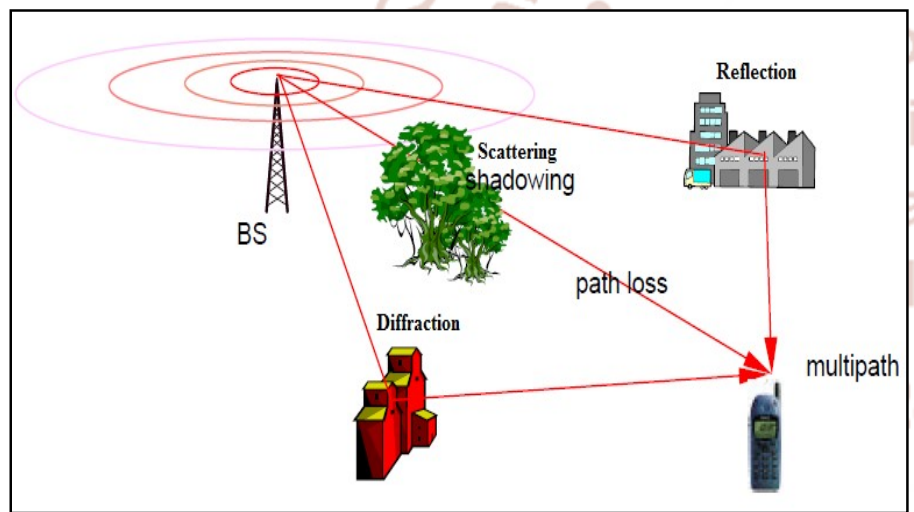

Fig. 2: NLOS Prorogation

\section{MIMO-System}

The two representations of space diversity techniques are transmit diversity where multiple transmit antennas are used (also known as multiple-input single-output or MISO systems) and receive ( $\mathrm{Rx})$ diversity where multiple receive antennas are used (also 5 known as single-input multiple-output or SIMO systems). In both cases it is required for antennas to be placed sufficiently far apart so that the channel gains between different antenna pairs fade independently. The achievable capacity and performance depend on the channel conditions and on the structure of the transmit signal. In order to achieve the goal the design MIMO system architecture influences the complexity of the transmitter and, particularly the receiver [4].

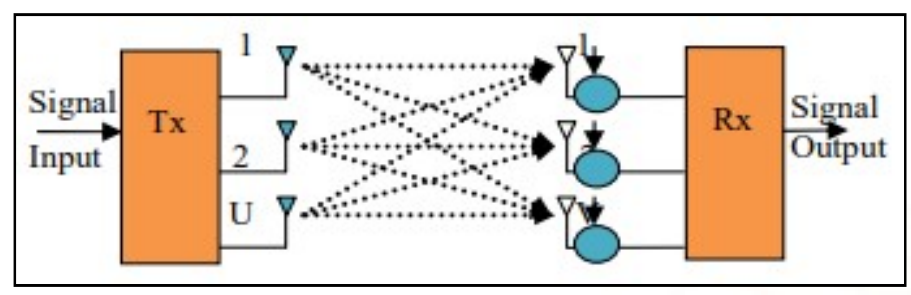

Fig. 3: MIMO Transceiver Structure [5]

\section{SIMULATION PARAMETERS}

In this work, we consider MMSE Equalizers techniques with MIMO system and following simulation parameters are considered for simulation of results as shown in table 1.

Table: 1 Simulation Parameters

\begin{tabular}{|c|c|}
\hline PARAMIETERS & VALUES \\
\hline Technology & V-BLAST \\
\hline Data Bits & $10^{6}$ \\
\hline Number of Transmitter & 2 \\
\hline Number of Receiver & 2 \\
\hline Algorithm & $\mathrm{ZF}$ and MMSE \\
\hline Modulation Techniques & BPSK \\
\hline Channel & Rayleigh, AWGN \\
\hline Simulation toll & Matlab R2013a \\
\hline Calculation Parameters & BER V/S SNR \\
\hline $\mathrm{X}$-axis & SNR in $\mathrm{dB}$ \\
\hline Y-axis & BER \\
\hline Technology & $\begin{array}{l}\text { Wireless } \\
\text { Communication }\end{array}$ \\
\hline
\end{tabular}

\section{SIMULATION RESULTS FOR MMSE EQUALIZERS}

In this section results are shown in terms of Bit Error Rate (BER) with respect to variation in SNR for $2 \mathrm{X} 2$ MIMO-V-BLAST system using BPSK modulation techniques and MMSE, MMSE-SIC and MMSE-SICSort equalisation algorithms under Rayleigh channels.

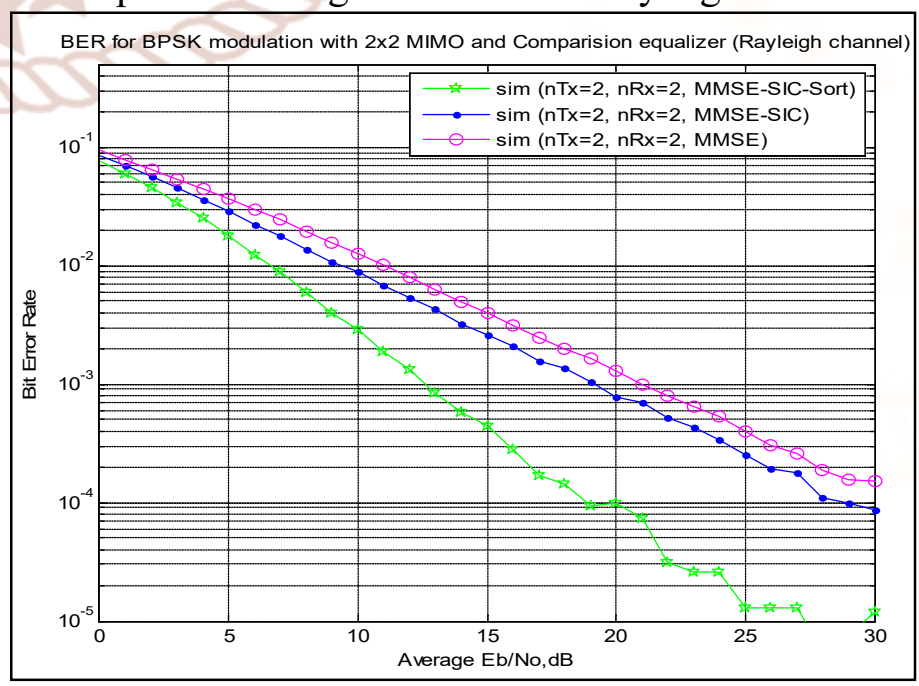

Fig. 3: Comparison Results for BPSK modulation with $2 \times 2$ MIMO with different MMSE 
The BER values have been computed as a function of SNR for BPSK modulation and different combinations of MIMO systems using MMSE, MMSE-SIC and Modified MMSE-SIC-Sort, and ML equalizers. The simulations studies have been carried out using MATLAB software. In the above result observed that MMSE-SIC-Sort has reduced error at all the point of SNR range 0 to $27 \mathrm{~dB}$.

\section{CONCLUSION}

The MIMO systems are gaining much more attention and efforts in wireless communication research due to their potential to increase considerable capacity in mobile cellular communication. The BER values have been computed as a function of SNR for BPSK modulation and different combinations of MIMO systems using MMSE, MMSE-SIC and Modified MMSE-SIC-Sort, and ML equalizers. The simulations studies have been carried out using MATLAB software. In the above result observed that MMSESIC-Sort has reduced error at all the point of SNR range 0 to $27 \mathrm{~dB}$.

\section{REFERENCES}

1. G. J. Foschini, "Layered Space-Time Architecture for Wireless Communication in a l"Fading Environment When Using Multiple Antennas," Bell Lab. Tech. J., v. 1, N. 2, pp. 41- 59, 1996.

2. G. J. Foschini, P. W. Wolnainsky, G. D. Golden, and R. A. Valenzuela, "Simplified Processing for High Spectral Efficiency Wireless Communication Employing Multi-Element Arrays," IEEE J. Select. Areas Commun., vol. 17, no. 11, pp. 1841-1852, Nov. 1999.

3. P. W. Wolnainsky, G. J. Foschini, G. D. Golden, and R. A. Valenzuela, "V-BLAST: An Architecture for Achieving Very High Data Rates Over the Rich-Scattering Wireless Channel," Int. Symposium on Signals, Systems and Electronics (ISSSE), Pisa, Italy, 1998.

4. Priya Sharma and Vijay Prakash Singh, Performance Evaluation of V-Blast MIMO
System in Fading Diversity Using Matched Filter, International Journal of Emerging Technology and Advanced Engineering, Vol. 3(5), 2013.

5. Sabuj Sarkar "An Advanced Detection Technique in MIMO--PSK Wireless Communication Systems Using MMSE-SIC Detection over a Rayleigh Fading Channel" Springer 2016.

6. Chandani Dewangan and Pankaj M Gulhane "Performance Evaluation of Different Equalization Techniques for $2 \times 2$ MIMO Wireless Communication Systems" International Journal of Advanced Research in Education \& Technology (IJARET), Vol. 3, Issue 3, 2016.

7. D. W. Tufts, "Nyquist's problem-The joint optimization of transmitter and receiver in pulse amplitude modulation," Proc. IEEE, vol. 53, pp. 248-260, Mar. 1965.

8. Anuj Kanchan, Shashank Dwivedi, "Comparison of BER Performance in OFDM Using Different Equalization Techniques", International Journal of Engineering and Advanced Technology (IJEAT) ISSN: $2249-8958$, Volume-1, Issue-6, August 2012, pp. 139-143.

9. Priyant Sharma and Vijay Prakash Singh, "Performance Evaluation of V-Blast MIMO System in Fading Diversity Using Matched Filter", International Journal of Emerging Technology and Advanced Engineering, Vol. 3(5). 2013.

10. Nirmalendu Bikas Sinha, S. Chakraborty, P. K. Sutradhar, R. Bera, and M. Mitra, "Optimization of MIMO Detectors: Unleashing the Multiplexing Gain" Journal of Telecommunication, Vol.1, 335342, Feb. 2011.

11. Samarendra Nath Sur, "Performance Analysis of V-BLAST MIMO System in Rician Channel Environment", Journal of Theoretical and Applied Information Technology, Vol. 2, 597-601, 2011. 